\title{
Temporal distribution and insecticide resistance profile of two major arbovirus vectors Aedes aegypti and Aedes albopictus in Yaoundé, the capital city of Cameroon
}

\author{
Basile Kamgang $^{1^{*}}$ D, Aurelie P. Yougang ${ }^{1,2}$, Micareme Tchoupo ${ }^{1}$, Jacob M. Riveron ${ }^{1,3}$ and Charles Wondji1,3
}

\begin{abstract}
Background: Aedes aegypti and Ae. albopictus are the major epidemic vectors of several arbovirus diseases such as yellow fever, dengue, Zika and chikungunya worldwide. Both Aedes vectors are presents in Cameroon; however, knowledge on the dynamic of the distribution of these species across cities and their resistance profile to insecticide are limited. Here, we assessed the current distribution of Ae. aegypti and Ae. albopictus in Yaoundé, the Capital City, established the resistance profile to insecticides and explored the resistance mechanisms involved.

Methods: Immature stages of Aedes were sampled in several breeding sites in December 2015 (dry season) and June 2016 (rainy season) in three central neighborhoods and four peripheral neighborhoods and reared to adult stage. The G0 adults were used for molecular identification and genotyping of F1534C mutation in Ae. aegypti. Bioassays and piperonyl butoxide (PBO) assays were carried out according to WHO guidelines.

Results: Analysis revealed that both species Ae. aegypti and Ae. albopictus are present in all prospected sites in Yaounde. However, in the dry season Ae. aegypti is most abundant in neighborhoods located in downtown. In contrast, Ae. albopictus was found most prevalent in suburbs whatever the season and in downtown during the rainy season. Bioassay analysis showed that both Ae. aegypti and Ae. albopictus, are resistant to $0.05 \%$ deltamethrin, $0.1 \%$ bendiocarb and $4 \%$ dichlorodiphenyltrichloroethane (DDT). A decreased of susceptibility to $0.75 \%$ permethrin and a full susceptibility to malathion $5 \%$ was observed. The mortality rate was increased after pre-exposure to synergist PBO. None of Ae. aegypti assayed revealed the presence of F1534C mutation.

Conclusion: These findings are useful to planning vector control programme against arbovirus vectors in Cameroon and can be used as baseline in Africa where data on Aedes resistance is very scarce to plan further works.
\end{abstract}

Keywords: Aedes aegypti, Aedes albopictus, Arboviruses, Temporal distribution, Spatial distribution, Insecticide resistance

\section{Background}

Yellow fever virus, dengue virus (DENV), chikungunya virus (CHIKV) and Zika virus (ZIKV) are mosquitoborne viruses of medical concern in tropical and subtropical regions. In Africa, until recently, the situation seemed to be of little concern because outbreaks of dengue without haemorrhagic syndromes had been observed

\footnotetext{
* Correspondence: kamgang_d@yahoo.fr

${ }^{1}$ Research Unit Liverpool School of Tropical Medicine/Organisation de Coordination pour la lutte contre les Endémies en Afrique Centrale, P.O. Box 288, Yaoundé, Cameroon

Full list of author information is available at the end of the article
}

only in East Africa [1, 2]. However, during the past decade, dengue outbreaks have been reported in several West and Central African countries [3-6] suggesting a possible change in the dynamic of this disease. Similarly, CHIKV, which previously caused only sporadic epidemics in sub-Saharan Africa [7], has recently emerged in several urban epidemic foci in Central Africa [6]. Formerly, sporadic isolation of ZIKV has been documented in human and mosquitoes in Asia and Africa [8]. But since the epidemic reported in Micronesia in 2007 [9], the geographical distribution has been 
expanded in the Americas where a massive outbreak has been reported [10].

Aedes aegypti Linneaus and Ae. albopictus (Skuse) are the main epidemic vectors of these viruses worldwide [11-13]. Both species are established in sub-Saharan Africa, where Ae. aegypti is native [14]. Aedes albopictus originated from Asia [15], has invaded all the five continents during the past three decades [16]. This species has been first reported in Central Africa in Cameroon in early 2000s, and since then has invaded almost all central African countries [6, 17]. Alarmingly, the introduction of Ae. albopictus in Central Africa coincided with the emergence of DENV, ZIKV and CHIKV in urban areas [6].

In Central Africa, Ae. aegypti and Ae. albopictus are found sympatric in several locations, notably in south Cameroon [18]. Both species are found in rural and urban areas where they breed in domestic (e.g. water storage and flower pots), peri-domestic (e.g. discarded tanks and used tyres) and natural (e.g. tree holes and plant axils) breeding sites $[17,18]$. As there is still no vaccine or specific treatment for these viruses, vector control remains the cornerstone of prevention and outbreak control. The conventional strategies for controlling Aedes species are based on reduction of breeding sites and on insecticide-based interventions. Indeed, in emergency situations, space spraying with adulticides can reduce the density of adult mosquitoes [19]. Unfortunately, many vector control programmes are threatened by the development of insecticide resistance in $A e$. aegypti and Ae. albopictus [20, 21]. Two major resistance mechanisms have been found involved on insecticide resistance: insensitivity target sites and increase in the rate of insecticide metabolism [22, 23]. Target site resistance is caused by mutations in target genes such as the voltage gated sodium channel (VGSC) causing knockdown resistance $(k d r)$, mutations in the acetylcholinesterase (Ace-1) gene and GABA receptors [23, 24]. One of the most important target site resistance for mosquitoes is $k d r$ as it confers resistance to both pyrethroids and dichlorodiphenyltrichloroethane (DDT). Several $k d r$ mutations have been identified in Ae. aegypti, and the association between the V1016G/I and the F1534C mutations and pyrethroid resistance has been established [25-27]. $k d r$ mutation is less prevalent in Ae. albopictus with only the mutation F1534C that has been detected [28]. However metabolic resistance through upregulation of detoxification genes is a major resistance mechanism in both species. The three main enzyme families responsible for insecticide resistance in mosquitoes are the monooxygenases (cytochrome P450s), glutathione Stransferases (GSTs) and carboxylesterases (COEs) [29, 30]. In Africa, data on ecological characterization of Aedes vectors as well as their insecticide resistance profiles are scarce.
In the context of emerging arboviruses in numerous countries across the world and in Africa particularly, this study was performed to assess the current spatio-temporal distribution of Ae. aegypti and Ae. albopictusin in Yaoundé, the capital city of Cameroon, and the insecticide resistance profile as well as exploring the resistance mechanism involved.

\section{Methods}

\section{Mosquito collection}

Immature stages of Ae. aegypti and Ae. albopictus were sampled in December 2015 (dry season) and June 2016 (rainy season) in seven neighborhoods in Yaoundé (Fig. 1). Based on a previous study in Yaoundé showing that Ae. albopictus seem to be most prevalent in peripheral areas of the city while Ae. aegypti is predominant in the city center [31], we selected three central neighborhoods (Mokolo, Mvog-Ada and Essos) and four peripheric (Nkolbisson, Emana, Ahala and Nkoabang). In each selected neighborhood, all potential larval breeding sites were inspected and positive sites (with at least one Aedes larvae or pupae) recorded. Immature stages of Aedes were collected, transported to the insectaries, pooled according to the location and reared to adult stage for identification. Adult mosquitoes were morphologically identified [32], numbered, pooled in a breeding cage according to species and location and further reared in the controlled condition $\left(27 \pm 2{ }^{\circ} \mathrm{C}\right.$; relative humidity $80 \pm 10 \%$ ) until generation 1 (G1) or subsequent G2 and G3. The comparison between the prevalence of $A e$. aegypti and Ae. albopictus has been performed using chi-square tests.

\section{Adult insecticide bioassays}

Aedes aegypti and Ae. albopictus bioassays were performed with non-blood-fed females according to the standard WHO guidelines [33]. Two reference susceptible strains were used as controls: the Ae. aegypti New Orleans strain and the Ae. albopictus susceptible strain from the Malaysia Vector Control Research Unit. Insecticide-impregnated papers were supplied by Liverpool School of Tropical Medicine. Two to five day-old F3 generation of Aedes mosquitoes from dry season and F1 generation from raining season with 4 replicates of 25 mosquitoes per tube were tested. The insecticides tested were: $0.75 \%$ permethrin (type I pyrethroid), $0.05 \%$ deltamethrin (type II pyrethroid), 4\% DDT (organochlorine), $0.1 \%$ bendiocarb (carbamate) and 5\% malathion (organophosphate). Mortality was recorded after $24 \mathrm{~h}$ and survivors were stored at $-80{ }^{\circ} \mathrm{C}$ whereas dead mosquitoes were kept in silica gel into $1.5 \mathrm{ml}$ tubes.

\section{Synergist assay with piperonyl butoxide}

In order to investigate the potential role of oxidasespecific metabolic resistance mechanisms, synergist assays 


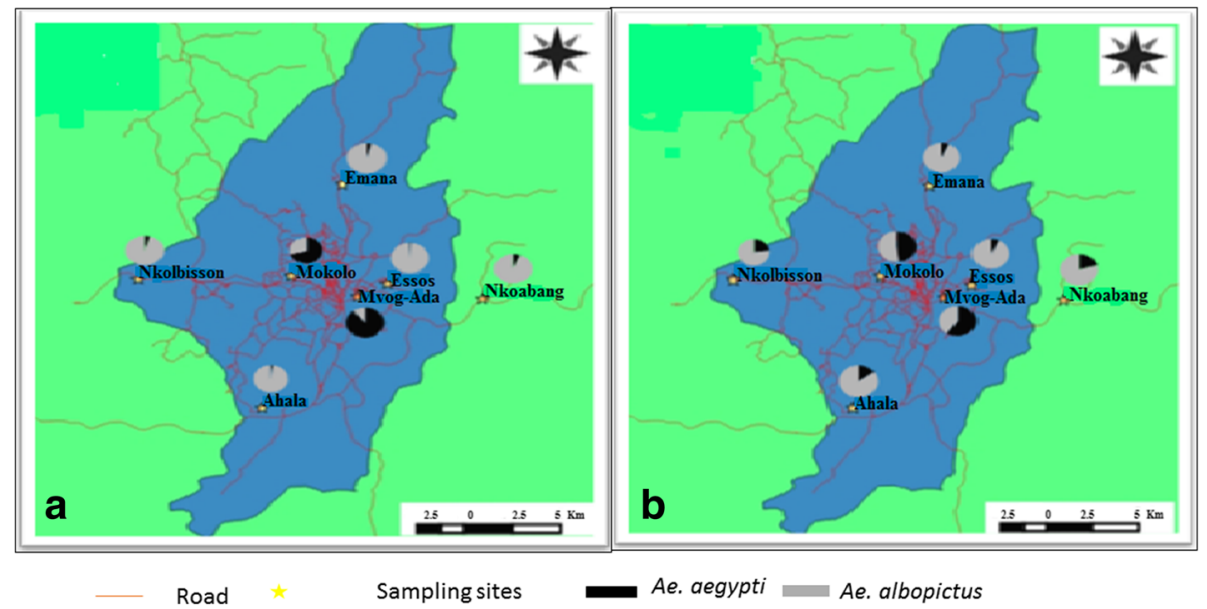

Fig. 1 Sampling sites and geographical distribution of Ae. aegypti and Ae. albopictus in Yaoundé according to the season. a Dry season. b Rainy season

with piperonyl butoxide (PBO) was performed. Adult 2-5 day-old mosquitoes were pre-exposed to papers impregnated with $4 \%$ PBO for one $h$ and then immediately exposed to three insecticides, DDT, deltamethrin and bendiocarb, for which higher level of resistance has been observed. Mortality was recorded after $24 \mathrm{~h}$ and compared to the results obtained with each insecticide without $\mathrm{PBO}$ and to a control sample exposed only to PBO.

\section{F1534C genotyping using allele specific PCR in Aedes aegypti}

Genomic DNA was extracted for 59 and 83 F0 individuals of Ae. aegypti and Ae. albopictus, respectively, from two locations, using the Livak method [34]. To confirm morphological identification, molecular identification was carried out using a polymerase chain reaction (PCR) based method described by Beebe et al. [35] to differentiate between Ae. aegypti and Ae. albopictus. All specimens confirmed as Ae. aegypti were used to genotype the F1534C mutation known to be associated to DDT and pyrethroid resistance in Ae. aegypti in several countries worldwide including Africa such as in Ghana (West Africa) [36]. This mutation was genotyped using the allele specific PCR method as previously described [37].

\section{Results}

Relative abundance and spatial distribution of Ae. aegypti and Ae. albopictus

A total of 4971 immature specimens of Aedes spp. were collected in December 2015 (dry season) and June 2016 (rainy season) in seven neighborhoods of the Yaoundé city. Aedes albopictus was found more prevalent (74.2\%) than Ae. aegypti (25.8\%). Analyses performed according to locations (suburbs vs downtown) and seasons revealed that in dry season Ae. aegypti is most abundant in neighborhoods located in downtown such as Mokolo and Mvog-Ada (Table 1). In contrast, Ae. albopictus was found most prevalent in suburbs whatever the season and in downtown during the rainy season (Table 1). No significant difference was found between overall numbers of Ae. albopictus and Ae. aegypti collected in downtown during the dry season $\left(\chi^{2}=2.25, d f=2, P>0.2\right)$, while the number of Ae. albopictus was significantly higher than those of Ae. aegypti $\left(\chi^{2}=1125.96, d f=2, P<0.001\right)$ in suburbs during the dry season and in rainy season irrespective to the location (Table 1). In suburbs, the number of $A e$. aegypti was lower than the number of Ae. albopictus in each location independently to the season (Table 1). In the other hand, in downtown the abundance of Ae. aegypti was higher than that of Ae. albopictus $\left(\chi^{2}=369.05, d f=2\right.$, $P<0.001)$ in Mokolo and $\left(\chi^{2}=240.25, \quad d f=2\right.$, $P<0.001)$ Mvog-Ada in the dry season whereas in the rainy season $A$ e. albopictus was more abundant than Ae. aegypti in Essos $\left(\chi^{2}=295.81, d f=2, \mathrm{P}<0.001\right)$. The assessment of the spatial distribution of these two species in Yaoundé showed that both Aedes species Ae. aegypti and Ae. albopictus coexist in all the prospected areas of the city (Fig. 1, Table 1).

\section{Insecticide resistance profiles}

Tests performed with laboratory strains confirmed that Ae. albopictus (VCRU) and Ae. aegypti (New Orleans) were totally susceptible to insecticides tested except to DDT for which $80.68 \%$ and $98.75 \%$ mortality rates were found, respectively. The mortality rate in controls was inferior to $5 \%$.

\section{Resistance pattern for Aedes aegypti}

The two populations collected during the dry season were first tested revealing that both were resistant to the type II pyrethroid, deltamethrin, particularly in females with similar mortality rates ranging from $82.42 \%$ in 
Table 1 Relative abundance of Ae. aegypti and Ae. albopictus from several locations in Yaoundé according to season

\begin{tabular}{|c|c|c|c|c|c|c|c|c|}
\hline \multirow[t]{2}{*}{ Location } & \multicolumn{4}{|l|}{ Dry season } & \multicolumn{4}{|l|}{ Rainy season } \\
\hline & Breeding sites $^{a}$ & Ae. aegypti & Ae. albopictus & $P$ & Breeding sites $^{a}$ & Ae. aegypti & Ae. albopictus & $P$ \\
\hline Downtown & 21 & 509 & 544 & $<0.1$ & 29 & 492 & 731 & $<0.001$ \\
\hline Mokolo & $\begin{array}{l}\text { Used tires (10); } \\
\text { Car wrecks (1) }\end{array}$ & 470 & 199 & $<0.001$ & Used tires (5) & 178 & 192 & $>0.05$ \\
\hline Mvog-ada & Used tires (2) & 35 & 4 & $<0.001$ & Used tires (11) & 289 & 195 & $<0.001$ \\
\hline Essos & Used tires (8) & 4 & 341 & $<0.001$ & $\begin{array}{l}\text { Used tires (12); } \\
\text { Discarded tanks (1) }\end{array}$ & 25 & 344 & $<0.001$ \\
\hline Surbub & 50 & 49 & 1222 & $<0.001$ & 78 & 232 & 1192 & $<0.001$ \\
\hline Emana & Used tires (8) & 10 & 241 & $<0.001$ & $\begin{array}{l}\text { Used tires (13); } \\
\text { Cobblestone moulds (30) }\end{array}$ & 37 & 491 & $<0.001$ \\
\hline Nkolbisson & Used tires (10) & 28 & 549 & $<0.001$ & $\begin{array}{l}\text { Used tires (9); } \\
\text { Discarded tanks (1); } \\
\text { Rubber boot (1) }\end{array}$ & 146 & 480 & $<0.001$ \\
\hline Ahala & $\begin{array}{l}\text { Used tires (10); } \\
\text { Discarded tanks (5) }\end{array}$ & 9 & 404 & $<0.001$ & Used tires (9) & 19 & 105 & $<0.001$ \\
\hline Nkoabang & Used tires (16) & 2 & 28 & $<0.001$ & Used tires (15) & 30 & 116 & $<0.001$ \\
\hline
\end{tabular}

${ }^{a}$ Numbers in parentheses indicate the number of breeding sites with immature stages of Aedes

Mokolo to $83.90 \%$ in Mvog-Ada (Additional fie 1: Table S1). In contrast, both populations were fully susceptible to type I pyrethroid, permethrin. Assays with the carbamate, bendiocarb, revealed that both populations were resistant to this insecticide particularly in females with similar mortality of 79.78 and $79.52 \%$ in Mokolo and Mvog Ada, respectively (Additional file 1: Table S1, Fig. 2). The highest level of resistance was observed against DDT with both populations resistant with mortality rates ranging from 19.57 to $36.47 \%$ in Mokolo and Mvog-Ada, respectively for females
(Additional file 1: Table S1, Fig. 2). However, both populations were fully susceptible to the organophosphate malathion.

Bioassays performed during the raining season were overall consistent with the results obtained during the dry season apart for DDT for which an increased mortality was observed in the raining season in Mokolo (19.57-57.65\%; $\left.\chi^{2}=25.1, d f=2, P<0.001\right)$ and Mvog-Ada (36.47-69.62\%; $\left.X^{2}=15.7, d f=2, P<0.001\right)$. The third population of Ahala tested in the wet season also showed

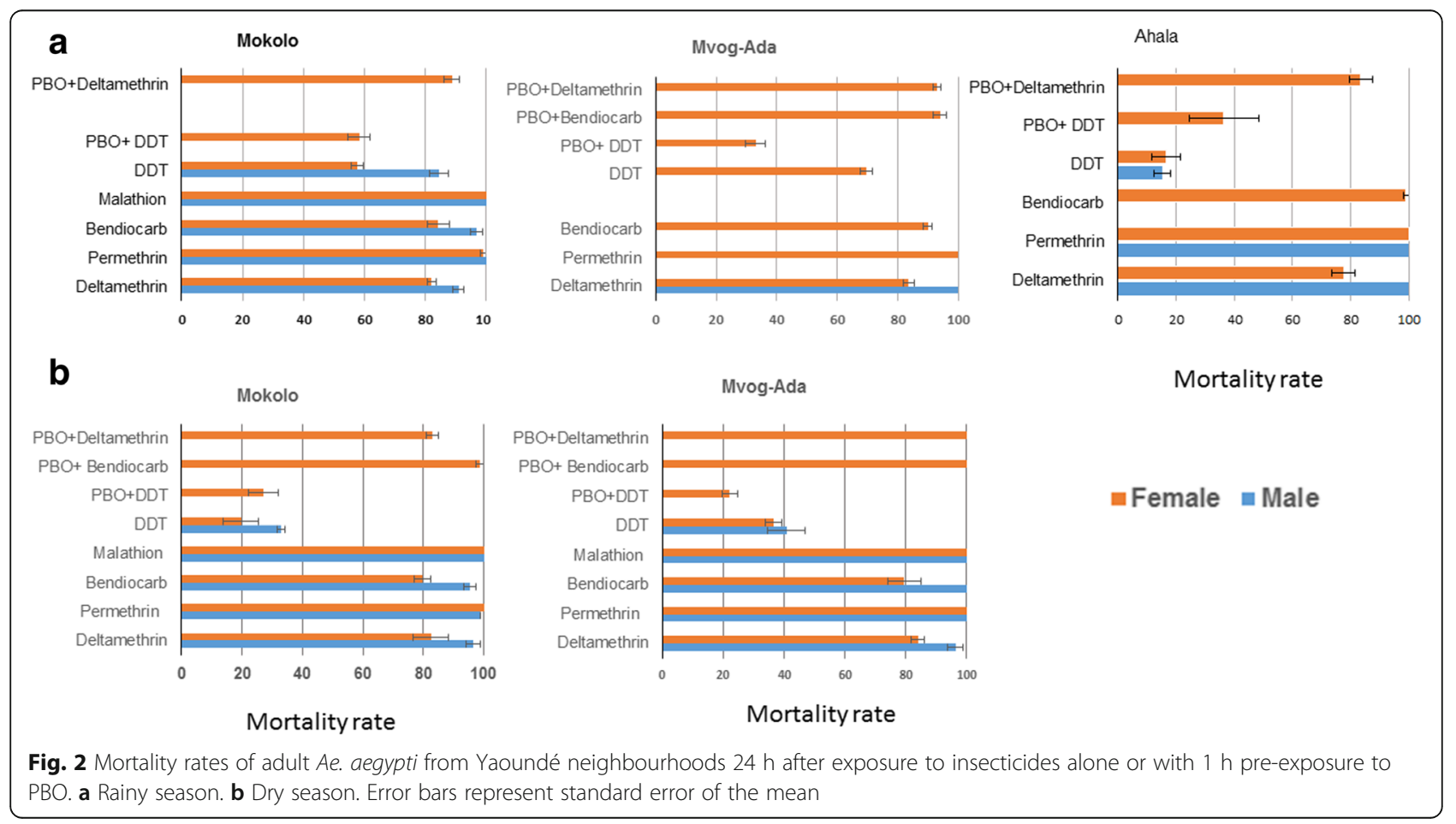


similar resistance profiles to the other two. However, the Ahala population was fully susceptible to bendiocarb and exhibited a greater resistance to DDT (16.48\%) than the other two populations (Additional file 1: Table S1, Fig. 2).

\section{Aedes albopictus resistance pattern}

Bioassays performed during the dry season revealed that three populations tested were resistant to the type II pyrethroid, deltamethrin although in Mokolo this was only moderate with $95.8 \%$ in females (Fig. 3, Additional file 2: Table S2). As for Ae. aegypti, a full susceptibility was observed against the type I permethrin and also against the organophosphate malathion in all populations. A moderate resistance was observed against the carbamate bendiocarb with mortality rates ranging between $93.1-95.5 \%$ in females. Also similar to Ae. aegypti, high resistance levels were observed against DDT in all three populations with mortality rates ranging between 5.9-44.8\% in females (Fig. 3, Additional file 2: Table S2).

The samples collected during the wet season exhibited a greater resistance levels for several insecticides (Fig. 4). This includes deltamethrin for which a decrease in mortality rates was observed in two populations from 95.8 to 69.3\% in Mokolo $\left(\chi^{2}=10.13, d f=2, P<0.005\right)$ and 78.3 to $55.2 \%$ in Emana $\left(\chi^{2}=9.66, d f=2, P<0.005\right.$ ) (Fig. 4 , Additional file 2: Table S2). Similarly, for bendiocarb, mortality rates decreased from 93.8 to $69.2 \%$ in Mokolo $\left(\chi^{2}=10.13, d f=2, P<0.005\right)$ and from 95.2 to $69 \%$ in
Emana $\left(\chi^{2}=9.94, d f=2, P<0.005\right)$ but no significant change was observed in Ahala. The Mokolo and Emana populations were resistant to permethrin in the wet season with mortality rates of 86 and $87.3 \%$, respectively. While $100 \%$ of mortality were found in dry season (Additional file 2: Table S2) event if no significant difference was observed on mortality rate between both seasons).

\section{Synergist assays with PBO}

Pre-exposure of mosquito samples to the PBO synergist before bioassays to deltamethrin, DDT and bendiocarb revealed contrasting results with both cases of increased or unchanged mortality rates in both Ae. aegypti (Additional file 1: Table S1) and Ae. albopictus (Additional file 2: Table S2) with deltamethrin and bendiocarb during both dry and wet seasons. One example is the case of the Ae. aegypti population from Emana during the dry season which showed a recovery of susceptibility from 83.9 to $100 \%$ after PBO exposure. However other populations did not show a significant increase in mortality after PBO such as the Ae. aegypti populations from Mokolo (82.4\% mortality without PBO and $82.6 \%$ after PBO exposure). On the other hand, no increase of mortality was observed with DDT in Ae. aegypti from Mvog-Ada (Additional file 1: Table S1) and Ae. albopictus from Mokolo and Ahala (Additional file 2: Table S2).

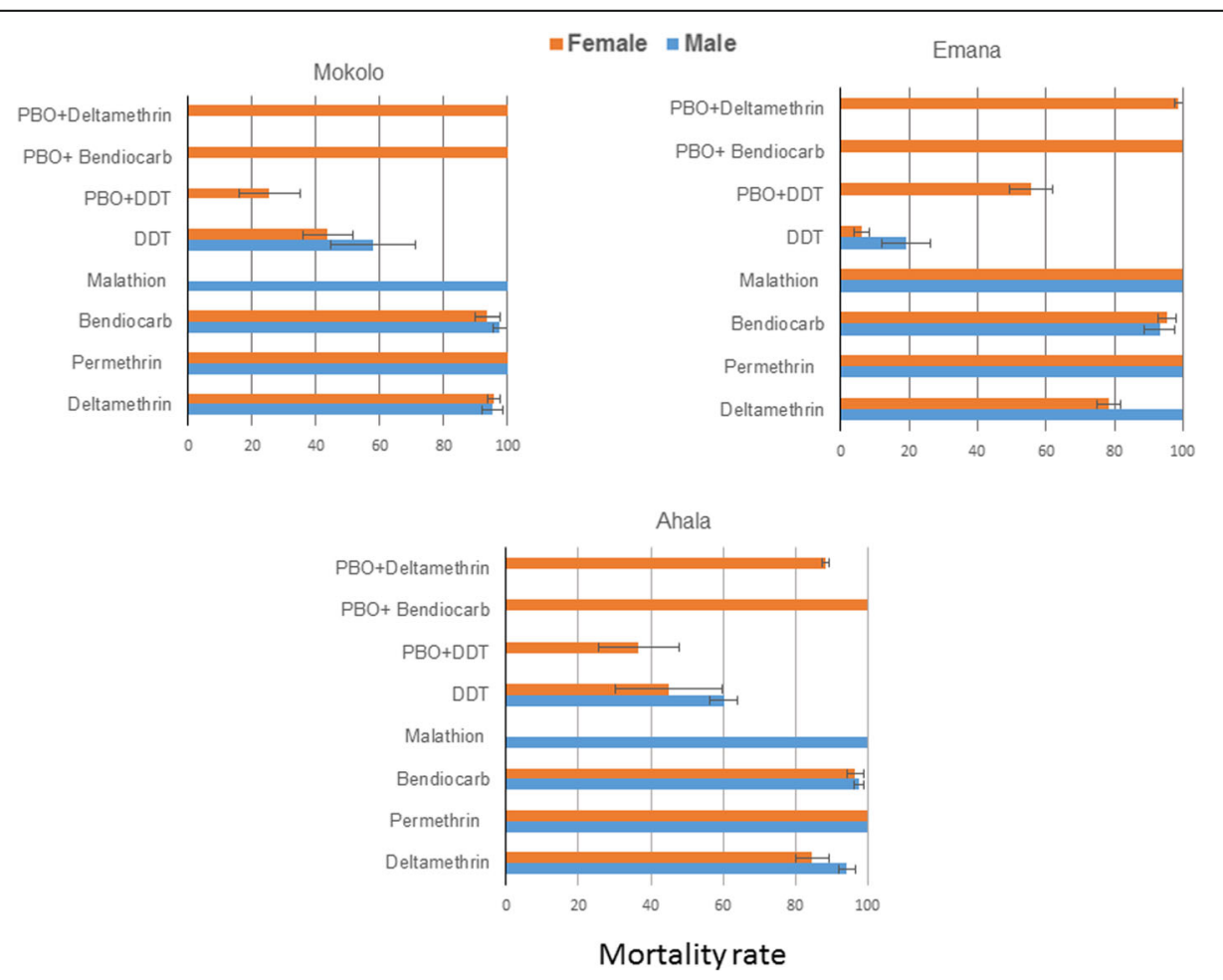

Fig. 3 Mortality rates of adult Ae. albopictus in the dry season from Yaoundé neighbourhoods $24 \mathrm{~h}$ after exposure to insecticides alone or with $1 \mathrm{~h}$ pre-exposure to PBO. Error bars represent standard error of the mean 

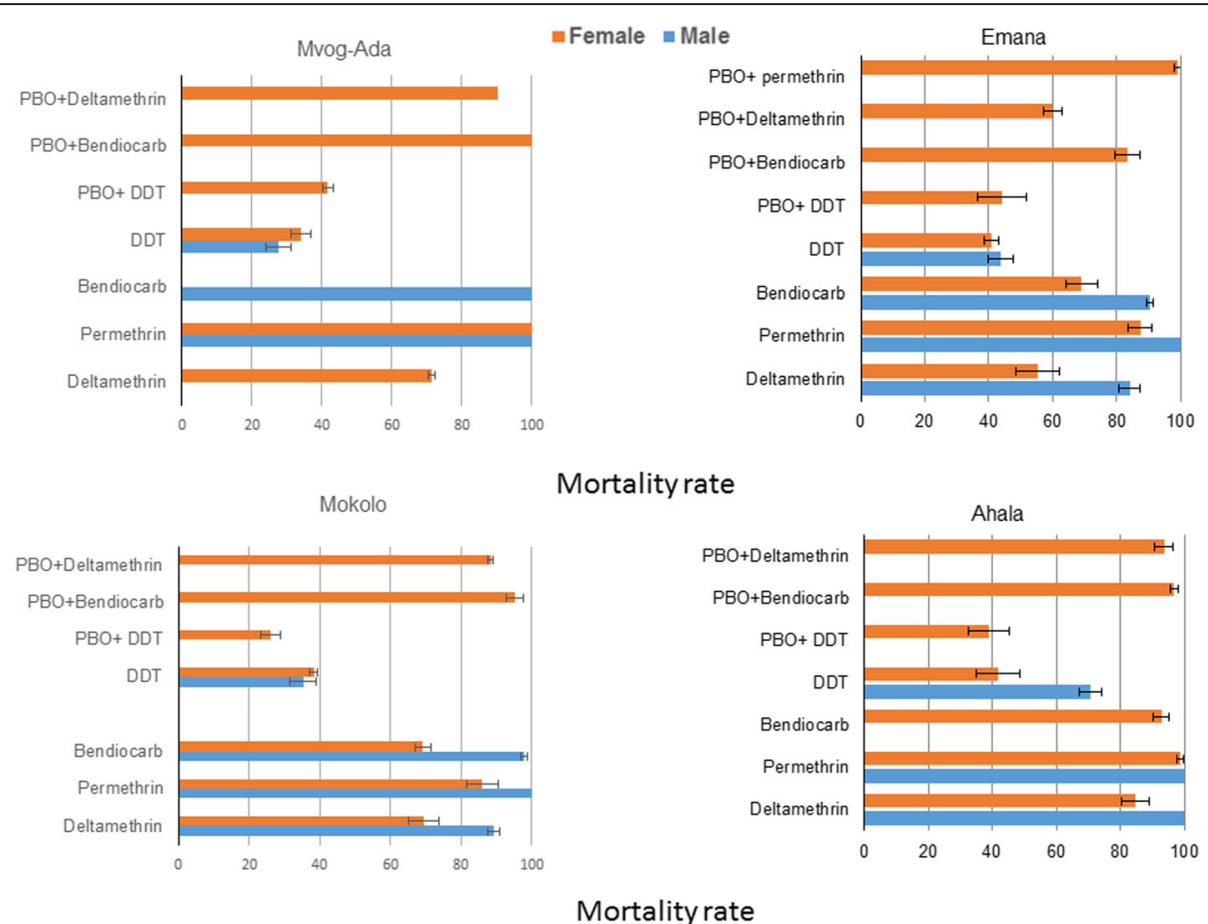

Fig. 4 Mortality rates of adult Ae. albopictus collected in the rainy season from Yaoundé neighbourhoods $24 \mathrm{~h}$ after exposure to insecticides alone or with $1 \mathrm{~h}$ pre-exposure to $\mathrm{PBO}$. Error bars represent standard error of the mean

\section{Genotyping of F1534C mutation in Aedes aegypti}

59 F0 specimens of Ae. aegypti and 83 F0 of Ae. albopictus identified morphologically were confirmed by molecular analysis. All the specimens of Ae. aegypti were used to search the presence of F1534C mutation. None of the Ae. aegypti mosquitoes was found carrying this mutation.

\section{Discussion}

This study presents the current distribution of $A e$. aegypti and Ae. albopictus in Yaoundé, the capital city of Cameroon and the resistance profile of these two species. The results revealed that both species are present in all the prospected locations of the city and Ae. albopictus is predominant during the two collection periods. Bioassay experiments revealed that both species are resistant to DDT and deltamethrin, loss of sensitivity was noticed to permethrin and bendiocarb, and fully susceptibility to malathion.

\section{Temporal distribution of both species}

The predominance of Ae. albopictus in both dry and rainy seasons is inconsistent with previous observations made in the Central African Republic [17] and in Florida, USA [38] showing the predominance of Ae. aegypti at the early wet season and Ae. albopictus in the late wet season. These findings had been explained by the higher tolerance of Ae. aegypti eggs than those of Ae. albopictus as demonstrated by Lounibos et al. [39].
The difference observed between these previous studies and the current is due probably to the difference of time between the rainy season and the dry season in these locations. The predominance of the invasive species $A e$. albopictus in Yaoundé is in agreement with the previous observations made in the city [31] suggesting that Ae. albopictus tends to supplant the indigenous species $A e$. aegypti. This observation suggests a good adaptation of Ae. albopictus due to the high ecological plasticity of this species, allowing it to adapt in different environments and to mating interference in sympatric areas as demonstrated by Bargielowski et al. [40]. Aedes aegypti was found dominant in the downtown particularly during the dry season such as Mokolo and Mvog-Ada whereas Ae. albopictus is the most abundant species in the suburbs. These findings are consistent with previous results showing segregation of both species according to urbanization in sympatric area [38]. Nevertheless, Ae. albopictus was found more abundant during the rainy season in Mokolo (downtown neighborhood). This difference between both seasons is probably due to the environmental change notably destroyed buildings due to regeneration project in Mokolo which has allowed the proliferation of vegetation which is very favourable to the development of Ae. albopictus as demonstrated previously [17, 31].

The higher number of immature stage of Aedes spp. collected in the rainy season is in agreement with 
previous observations showing that rainy season corresponds to the period in which the maximum densities of Aedes spp. mosquitoes are observed [17], suggesting higher risk of arboviruses transmission. However, in some neighborhoods fewer mosquitoes were collected in the wet season, such as the case of Ahala and Mokolo, potentially due to water overflow from some breeding sites which could have drained larvae from their habitats.

\section{Insecticide resistance patterns between both species}

Overall, this study revealed that both species of Aedes present a similar resistance profile to main insecticides used in public health. Indeed, both species are resistant to $0.05 \%$ deltamethrin, $0.1 \%$ bendiocarb (carbamate) and $4 \%$ DDT and fully susceptible to 5\% malathion. Almost all samples were found susceptible to $0.75 \%$ permethrin except two samples from Mokolo and Emana collected during the rainy season. This result is different to a previous study assessing the insecticide resistance profile of Ae. aegypti and Ae. albopictus in four cities of Cameroon [41]. This previous study had revealed that Ae. aegyti from Yaoundé was fully susceptible to four insecticides tested (deltamethrin, DDT, propoxur (carbamate) and fenitrothion (organophosphate) while Ae. albopictus was resistant to deltamethrin and DDT suggesting that both species have developed resistance to most of these insecticide classes in the past five years. However, a striking difference was observed with pyrethroids with higher resistance in deltamethrin (type II) whereas both species were more susceptible to permethrin (type I). Such differences have been previously observed in other populations of mosquitoes such as in Malaysia where an Ae. aegypti population from Kota Bharu was highly resistant to permethrin but not to deltamethrin [42, 43]. On the other hand, the low level of resistance reported to permethrin in these Aedes spp. populations could also be explained by the fact that the dose used in this study $(0.75 \%)$ is three-fold higher than what is recommended for Aedes species (0.25\%) [44]. It will be necessary to test these populations with this lower concentration of permethrin to establish the real resistance level. The high level of resistance observed in Ae. albopictus compared to that of Ae. aegypti is different from previous findings showing that Ae. aegypti is more resistant to pyrethroids than Ae. albopictus [42, 45]. Nevertheless, similar observations have been reported in previous studies in Central Africa, particularly in Cameroon [41] and the Central African Republic [46]. The decreased susceptibility to both types of pyrethroids observed in both populations may represent a serious threat for vector control programmes, since pyrethroids only are recommended for the control of adult Aedes mosquitoes notably in emergency situations [47, 48]. A loss of sensitivity was observed to bendiocarb for both species. Similar results have been observed previously in Pakistan and Malaysia $[42,49]$.

Both Ae. aegypti and Ae. albopictus samples from all locations were found resistant to DDT. Previous study in 1972 has reported decreased susceptibility to DDT in Ae. aegypti sampled in Yaoundé [50], suggesting continuing selection pressure on Aedes spp. populations. DDT-resistance to Ae. albopictus has also been reported in Yaoundé samples [41]. High DDT resistance in $A e$. aegypti [36, 42, 46, 51] and Ae. albopictus [41, 42, 45, 52] is commonly reported across the world. The higher level of DDT resistance in both species in Yaoundé is also similar to high resistance level to DDT observed in Cameroon in malaria vectors such as Anopheles gambiae [53] or An. funestus [54] probably as a consequence of the intense DDT spraying in the 1950s and 1960s as part of the malaria elimination campaign.

The causes of the resistance to pyrethroids and to bendiocarb in both species remains unclear since no specific vector control interventions targeting $A e$. aegypti and Ae. albopictus has been deployed [41]. Nevertheless, it is possible that insecticides used to control other insects of medical or agricultural interest exert indirect selection pressure on these two mosquito species as suggested previously [41, 46]. For Ae. albopictus, which was reported for the first time in Cameroon in the early 2000s, we cannot exclude the possibility that the invading population possessed a resistance background as suggested previously [41]. A temporal variation of the resistance profile was observed with high resistance during the second collection (rainy season) especially in Ae. albopictus samples particularly from Mokolo and Emana. The increasing of the level of resistance in the rainy season remains unclear.

Increasing of mortality rates in both Ae. aegypti and Ae. albopictus with deltamethrin and bendiocarb after pre-exposure to $\mathrm{PBO}$ suggest that cytochrome $\mathrm{P} 450$ monooxygenases are playing a predominant role in the observed resistance. On the other hand, no increase of mortality observed with DDT in both species in certain locations and no full recovery of susceptibility suggest that other enzymes such as GSTs may also implicated. None of the specimens of $A e$. aegypti genotyped was found with the presence F1534C mutation suggesting this mutation is not involved in resistance in the sample of Ae. aegypti tested. Nevertheless, this mutation was detected recently in Ae. aegypti sample from West Africa (Ghana) [36]. It will be interesting to extent this work in other locations throughout the country and also genotype other mutations such as $\mathrm{I} 1011 \mathrm{M} / \mathrm{V}$, V1016G/I which have been found involved in $k d r$ resistance in Ae. aegypti [25-27]. 


\section{Conclusion}

This study has shown that the invasive Ae. albopictus is now the most dominant arbovirus vector in Yaoundé irrespective of the season. It was also found this species to be more resistant than the indigenous species Ae. aegypti. This could be a concern for the control of arboviruses as Ae. albopictus has been reported to be mostly involved in the recent dengue, Zika and chikungunya outbreaks in Central Africa. Overall, the findings of this study highlight the need for more studies nationwide to better characterize these arbovirus vectors and help prepare potential outbreaks.

\section{Additional files}

Additional file 1: Table S1. Mortality rates of adult Ae. aegypti from Yaoundé neighbourhoods $24 \mathrm{~h}$ after exposure to insecticides alone or with $1 \mathrm{~h}$ pre-exposure to PBO. (DOC $47 \mathrm{~kb}$ )

Additional file 2: Table S2. Mortality rates of adult Ae. albopictus from Yaoundé neighbourhoods $24 \mathrm{~h}$ after exposure to insecticides alone or with $1 \mathrm{~h}$ pre-exposure to PBO. (DOC $52 \mathrm{~kb}$ )

\section{Abbreviations}

DDT: dichlorodiphenyltrichloroethane; $k d r$ : knock down resistance gene; PBO: piperonyl butoxide; PCR: polymerase chain reaction

\section{Acknowledgements}

We thank the population of Yaoundé for their collaboration during filed investigation.

\section{Funding}

This work was supported by the LSTM/OCEAC research unit through the Wellcome Trust 083515/Z/07/Z awarded to Charles Wondji. The funders had no role in study design, data collection or analysis, decision to publish or preparation of the manuscript.

\section{Availability of data and materials}

The dataset supporting the conclusions of this article is included within the article and its additional file.

\section{Authors' contributions}

BK and CW conceived and designed the experiments. BK, APY and TM performed the experiments. BK, APY and CW analysed the data. BK, JMR and CW contributed reagents, materials and analysis tools. BK, JMR and WC wrote the paper. All authors read and approved the final manuscript.

\section{Ethics approval and consent to participate}

Not applicable.

\section{Consent for publication}

Not applicable.

\section{Competing interests}

The authors declare that they have no competing interests.

\section{Publisher's Note}

Springer Nature remains neutral with regard to jurisdictional claims in published maps and institutional affiliations.

\section{Author details}

${ }^{1}$ Research Unit Liverpool School of Tropical Medicine/Organisation de Coordination pour la lutte contre les Endémies en Afrique Centrale, P.O. Box 288, Yaoundé, Cameroon. ${ }^{2}$ Department of Animal Biology, Faculty of Sciences, University of Yaoundé I, Yaoundé, Cameroon. ${ }^{3}$ Liverpool School of Tropical Medicine, Pembroke place, Liverpool L3 5QA, UK.
Received: 24 July 2017 Accepted: 26 September 2017

Published online: 10 October 2017

\section{References}

1. Kanesa-thasan N, Chang GJ, Smoak BL, Magill A, Burrous MJ. Hoke CHJr. Molecular and epidemiologic analysis of dengue virus isolates from Somalia. Emerg Infect Dis. 1998;4(2):299-303.

2. Rodier GR, Gubler DJ, Cope SE, Cropp CB, Soliman AK, Polycarpe D, et al. Epidemic dengue 2 in the city of Djibouti 1991-1992. Trans R Soc Trop Med Hyg. 1996;90(3):237-40.

3. Ridde V, Agier I, Bonnet E, Carabali M, Dabire KR, Fournet F, et al. Presence of three dengue serotypes in Ouagadougou (Burkina Faso): research and public health implications. Infect Dis Poverty. 2016:5:23.

4. Schwartz E, Meltzer E, Mendelson M, Tooke A, Steiner F, Gautret P, et al. Detection on four continents of dengue fever cases related to an ongoing outbreak in Luanda, Angola, March to May 2013. Euro Surveill. 2013;18(21)

5. Franco L, Di Caro A, Carletti F, Vapalahti O, Renaudat C, Zeller H, et al. Recent expansion of dengue virus serotype 3 in West Africa. Euro Surveill. 2010;15(7)

6. Ngoagouni C, Kamgang B, Nakoune E, Paupy C, Kazanji M. Invasion of Aedes albopictus (Diptera: Culicidae) into central Africa: what consequences for emerging diseases? Parasit Vectors. 2015:8:191

7. Diallo M, Thonnon J, Traore-Lamizana M, Fontenille D. Vectors of chikungunya virus in Senegal: current data and transmission cycles. Am J Trop Med Hyg. 1999;60(2):281-6

8. Haddow AD, Schuh AJ, Yasuda CY, Kasper MR, Heang V, et al. Genetic characterization of Zika virus strains: geographic expansion of the Asian lineage. PLoS Negl Trop Dis. 2012;6(2):e1477.

9. Duffy MR, Chen TH, Hancock WT, Powers AM, Kool JL, Lanciotti RS, et al. Zika virus outbreak on Yap Island, Federated States of Micronesia. N Engl J Med. 2009:360(24):2536-43.

10. Hennessey M, Fischer M, Staples JE. Zika virus spreads to new areas - Region of the Americas, May 2015 - January 2016. Am J Transplant. 2016;16(3):1031-4.

11. Paupy C, Ollomo B, Kamgang B, Moutailler S, Rousset D, Demanou M, et al. Comparative role of Aedes albopictus and Aedes aegypti in the emergence of dengue and chikungunya in central Africa. Vector Borne Zoonotic Dis. 2010; 10(3):259-66.

12. Grard G, Caron M, Mombo IM, Nkoghe D, Mboui Ondo S, Jiolle D, et al. Zika virus in Gabon (Central Africa) - 2007: a new threat from Aedes albopictus? PLoS Negl Trop Dis. 2014;8(2):e2681.

13. Wong SS, Poon RW, Wong SC. Zika virus infection-the next wave after dengue? J Formos Med Assoc. 2016;115(4):226-42.

14. Mattingly PF. Genetical aspects of the Aedes aegypti problem. I. Taxonomy and bionomics. Ann Trop Med Parasitol. 1957:51(4):392-408.

15. Gratz NG. Critical review of the vector status of Aedes albopictus. Med Vet Entomol. 2004;18(3):215-27.

16. Paupy C, Delatte H, Bagny L, Corbel V, Fontenille D. Aedes albopictus, an arbovirus vector: from the darkness to the light. Microbes Infect. 2009; 11(14-15):1177-85.

17. Kamgang B, Ngoagouni C, Manirakiza A, Nakoune E, Paupy C. Kazanji M Temporal patterns of abundance of Aedes aegypti and Aedes albopictus (Diptera: Culicidae) and mitochondrial DNA analysis of Ae. albopictus in the Central African Republic. PLoS Negl Trop Dis. 2013;7(12):e2590.

18. Simard F, Nchoutpouen E, Toto JC, Fontenille D. Geographic distribution and breeding site preference of Aedes albopictus and Aedes aegypti (Diptera: culicidae) in Cameroon, Central Africa. J Med Entomol. 2005;42(5):726-31.

19. WHO. WHO statement on the first meeting of the International Health Regulations. (IHR 2005) Emergency Committee on Zika virus and observed increase in neurological disorders and neonatal malformations. Geneva: WHO; 2005. p. 2016. http://www.who.int/mediacentre/news/statements/ 2016/1st-emergency-committee-zika/en/.

20. Marcombe S, Carron A, Darriet F, Etienne M, Agnew P, Tolosa M, et al. Reduced efficacy of pyrethroid space sprays for dengue control in an area of Martinique with pyrethroid resistance. Am J Trop Med Hyg. 2009;80(5):745-51.

21. Ranson H, Burhani J, Lumjuan N, Black WC. Insecticide resistance in dengue vectors. TroplKAnet. 2010;1(1):277-84

22. Labbé P, Alout H, Djogbénou L, Pasteur N, Weill M. 14. Evolution of resistance to insecticide in disease vectors. In: Tibayrenc $M$, editor. Genetics and Evolution of Infectious Disease. Elsevier; 2011. p. 363-409.

23. Hemingway J, Ranson $\mathrm{H}$. Insecticide resistance in insect vectors of human disease. Annu Rev Entomol. 2000;45:371-91. 
24. Sathantriphop S, Paeporn P, Supaphathom K. Detection of insecticides resistance status in Culex quinquefasciatus and Aedes aegypti to four major groups of insecticides. Trop Biomed. 2006;23(1):97-101.

25. Brengues C, Hawkes NJ, Chandre F, McCarroll L, Duchon S, Guillet P, et al. Pyrethroid and DDT cross-resistance in Aedes aegypti is correlated with novel mutations in the voltage-gated sodium channel gene. Med Vet Entomol. 2003;17(1):87-94

26. Yanola J, Somboon P, Walton C, Nachaiwieng W, Somwang P, Prapanthadara LA. High-throughput assays for detection of the F1534C mutation in the voltage-gated sodium channel gene in permethrin-resistant Aedes aegypti and the distribution of this mutation throughout Thailand. Tropical Med Int Health. 2011;16(4):501-9.

27. Saavedra-Rodriguez K, Urdaneta-Marquez L, Rajatileka S, Moulton M, Flores $A E$, Fernandez-Salas I, et al. A mutation in the voltage-gated sodium channel gene associated with pyrethroid resistance in Latin American Aedes aegypti. Insect Mol Biol. 2007;16(6):785-98.

28. Kasai S, Ng LC, Lam-Phua SG, Tang CS, Itokawa K, Komagata O, et al. First detection of a putative knockdown resistance gene in major mosquito vector, Aedes albopictus. Jpn J Infect Dis. 2011;64(3):217-21.

29. Li X, Schuler MA, Berenbaum MR. Molecular mechanisms of metabolic resistance to synthetic and natural xenobiotics. Annu Rev Entomol. 2007:52:231-53.

30. Perry T, Batterham P, Daborn PJ. The biology of insecticidal activity and resistance. Insect Biochem Molec. 2011:41(7):411-22.

31. Kamgang B, Happi JY, Boisier P, Njiokou F, Herve JP, Simard F, et al. Geographic and ecological distribution of the dengue and chikungunya virus vectors Aedes aegypti and Aedes albopictus in three major Cameroonian towns. Med Vet Entomol. 2010;24(2):132-41.

32. Jupp PG. Mosquitoes of Southern Africa: Culicinae and Toxorhynchitinae. Ekogilde Publishers: Hartebeespoort, Republic of South Africa; 1996.

33. WHO. Test procedures for insecticide resistance monitoring in malaria vector mosquitoes. Geneva: WHO; 2013

34. Livak KJ. Organization and mapping of a sequence on the drosophilamelanogaster $x$-chromosome and $y$-chromosome that is transcribed during spermatogenesis. Genetics. 1984;107(4):611-34.

35. Beebe NW, Whelan PI, Van den Hurk AF, Ritchie SA, Corcoran S, Cooper RD. A polymerase chain reaction-based diagnostic to identify larvae and eggs of container mosquito species from the Australian region. J Med Entomol. 2007:44(2):376-80.

36. Kawada H, Higa Y, Futami K, Muranami Y, Kawashima E, Osei JH, et al. Discovery of point mutations in the voltage-gated sodium channel from African Aedes aegypti populations: potential phylogenetic reasons for gene introgression. PLoS Negl Trop Dis. 2016;10(6):e0004780.

37. Harris AF, Rajatileka S, Ranson H. Pyrethroid resistance in Aedes aegypti from Grand Cayman. Am J Trop Med Hyg. 2010;83(2):277-84.

38. Braks MA, Honorio NA, Lourencqo-De-Oliveira R, Juliano SA, Lounibos LP. Convergent habitat segregation of Aedes aegypti and Aedes albopictus (Diptera: Culicidae) in southeastern Brazil and Florida. J Med Entomol. 2003; 40(6):785-94.

39. Lounibos LP, Suarez S, Menendez Z, Nishimura N, Escher RL, O'Connell SM, et al. Does temperature affect the outcome of larval competition between Aedes aegypti and Aedes albopictus? J Vector Ecol. 2002;27(1):86-95.

40. Bargielowski IE, Lounibos LP, Carrasquilla MC. Evolution of resistance to satyrization through reproductive character displacement in populations of invasive dengue vectors. Proc Natl Acad Sci USA. 2013;110(8):2888-92.

41. Kamgang B, Marcombe S, Chandre F, Nchoutpouen E, Nwane P, Etang J, et al. Insecticide susceptibility of Aedes aegypti and Aedes albopictus in Central Africa. Parasit Vectors. 2011:4:79.

42. Ishak IH, Jaal Z, Ranson H, Wondji CS. Contrasting patterns of insecticide resistance and knockdown resistance $(k d r)$ in the dengue vectors Aedes aegypti and Aedes albopictus from Malaysia. Parasit Vectors. 2015;8:181.

43. Ishak IH, Kamgang B, Ibrahim SS, Riveron JM, Irving H, Wondji CS. Pyrethroid resistance in Malaysian populations of dengue vector Aedes aegypti is mediated by CYP9 family of cytochrome P450 genes. PLoS Negl Trop Dis. 2017;11(1):e0005302

44. WHO. Entomological surveillance for Aedes spp. in the context of Zika virus: interim guidance for entomologists. Geneva: WHO; 2016.

45. Vontas J, Kioulos E, Pavlidi N, Morou E, della Torre A, Ranson H. Insecticide resistance in the major dengue vectors Aedes albopictus and Aedes aegypti. Pestic Biochem Physiol. 2012;104:126-31.

46. Ngoagouni C, Kamgang B, Brengues C, Yahouedo G, Paupy C, Nakoune E, et al. Susceptibility profile and metabolic mechanisms involved in Aedes aegypti and Aedes albopictus resistant to DDT and deltamethrin in the Central African Republic. Parasit Vectors. 2016:9(1):599.

47. Jirakanjanakit $N$, Rongnoparut $P$, Saengtharatip $S$, Chareonviriyaphap $T$, Duchon S, Bellec C, et al. Insecticide susceptible/resistance status in Aedes (Stegomyia) aegypti and Aedes (Stegomyia) albopictus (Diptera: Culicidae) in Thailand during 2003-2005. J Econ Entomol. 2007;100(2):545-50.

48. Macoris M, Andrighella M, Wanderley D, Ribolla P. Impact of insecticide resistance on the field control of Aedes aegypti in the State of Sao Paulo. Rev Soc Bras Med Trop. 2014;47:573-8.

49. Mohsin M, Naz SI, Khan I, Jabeen A, Bilal H, Ahmad R, et al. Susceptibility status of Aedes aegypti and Aedes albopictus against insecticides at eastern Punjab, Pakistan. MMJ. 2016;3(5):41-6.

50. Mouchet J, Cordellier R, Germain M, Carnevale P, Barathe J, Sannier C. Résistance aux insecticides d'Aedes aegypti et Culex pipiens fatigans en Afrique Centrale. Geneva: WHO/VBC/72/381; 1972. p. 12P.

51. Ayorinde A, Oboh B, Oduola A, Otubanjo O. The insecticide susceptibility status of Aedes aegypti (Diptera: Culicidae) in farm and nonfarm sites of Lagos State, Nigeria. J Insect Sci. 2015;15(1):75.

52. Marcombe S, Farajollahi A, Healy SP, Clark GG, Fonseca DM. Insecticide resistance status of United States populations of Aedes albopictus and mechanisms involved. PLoS One. 2014;9(7):e101992.

53. Fossog Tene B, Poupardin R, Costantini C, Awono-Ambene P, Wondji CS, Ranson $\mathrm{H}$, et al. Resistance to DDT in an urban setting: common mechanisms implicated in both $\mathrm{M}$ and $\mathrm{S}$ forms of Anopheles gambiae in the city of Yaounde Cameroon. PLoS One. 2013;8(4):e61408.

54. Menze BD, Riveron JM, Ibrahim SS, Irving H, Antonio-Nkondjio C, AwonoAmbene $\mathrm{PH}$, et al. Multiple insecticide resistance in the malaria vector Anopheles funestus from northern Cameroon is mediated by metabolic resistance alongside potential target site insensitivity mutations. PLoS One. 2016;11(10):e0163261.

\section{Submit your next manuscript to BioMed Central and we will help you at every step:}

- We accept pre-submission inquiries

- Our selector tool helps you to find the most relevant journal

- We provide round the clock customer support

- Convenient online submission

- Thorough peer review

- Inclusion in PubMed and all major indexing services

- Maximum visibility for your research

Submit your manuscript at www.biomedcentral.com/submit

) Biomed Central 